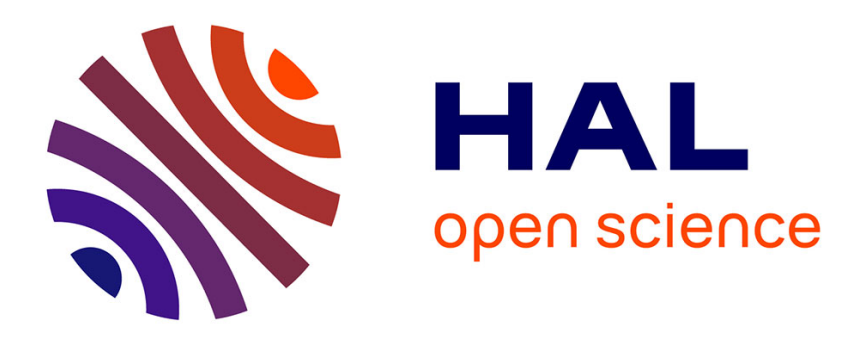

\title{
Nouveau dispositif d'étanchéité pour hautes pressions
}

\author{
J. Paureau
}

\section{To cite this version:}

J. Paureau. Nouveau dispositif d'étanchéité pour hautes pressions. Revue de Physique Appliquée, 1975, 10 (6), pp.475-478. 10.1051/rphysap:01975001006047500 . jpa-00243948

\section{HAL Id: jpa-00243948 https://hal.science/jpa-00243948}

Submitted on 1 Jan 1975

HAL is a multi-disciplinary open access archive for the deposit and dissemination of scientific research documents, whether they are published or not. The documents may come from teaching and research institutions in France or abroad, or from public or private research centers.
L'archive ouverte pluridisciplinaire HAL, est destinée au dépôt et à la diffusion de documents scientifiques de niveau recherche, publiés ou non, émanant des établissements d'enseignement et de recherche français ou étrangers, des laboratoires publics ou privés. 
Classification
Physics Abstracts

0.630

\title{
NOUVEAU DISPOSITIF D'ÉTANCHÉITÉ POUR HAUTES PRESSIONS
}

\author{
J. PAUREAU \\ Service National des Champs Intenses \\ C. N. R. S., BP 166, 38042 Grenoble Cedex, France
}

(Reçu le 3 mars 1975, révisé le 17 juin 1975, accepté le 19 juin 1975)

\begin{abstract}
Résumé. - On décrit et on donne les performances d'un nouveau dispositif d'étanchéité pour hautes pressions.

Abstract. - The description and the performances of a new high pressure sealing device are given.
\end{abstract}

1. Introduction. - Au cours de ces dernières années, les expériences de physique sous pression à basse température ont connu un certain développement [1-3]. Dans ces expériences, l'hélium constitue le milieu le mieux approprié pour transmettre la pression, en raison de l'étendue du domaine où il est fluide [4]. Mais les difficultés pratiques rencontrées pour réaliser un système d'étanchéité fiable n'ont rien de comparable avec celles rencontrées pour obtenir le même résultat avec un liquide sous pression. Les solutions adoptées en général, sont celles proposées par Bridgman [5] (Fig. $1 a$ et $b$ ). Chacune de ces solutions présente un certain nombre d'inconvénients. Dans la solution de la figure $1 a$, les joints exercent sur les portées une surpression qui au-delà d'une certaine valeur, provoque la striction de l'obturateur ; en outre lorsque ce dernier est prolongé par un tube capillaire, le remplacement des joints présente des difficultés. Dans la solution de la figure $1 b$, ces deux inconvénients ne se rencontrent pas, mais l'étanchéité aux gaz est aléatoire. Ces deux types de dispositif présentent deux autres inconvénients. D'une part, ils nécessitent l'utilisation-de plusieurs joints ; d'autre part, ils réclament un serrage énergique de ceux-ci pour initier l'étanchéité. Les auteurs de la référence [6] ont tenté d'améliorer le système de la figure $1 b$ en le dotant d'un obturateur à portée tronconique terminée par un congé. Retenant le principe de l'obturateur conique, nous avons mis au point un dispositif nouveau dont la description fait l'objet de cet article. Au paragraphe suivant, nous présenterons l'installation expérimentale. Dans le troisième paragraphe, nous préciserons les essais réalisés et nous indiquerons la démarche nous ayant permis d'aboutir au dispositif d'étanchéité dans sa forme finale. Enfin, nous en donnerons les performances.
2. Dispositif expérimental. - Le dispositif expérimental est constitué d'une enceinte d'essais en acier maraging reliée, par un tube capillaire, à un groupe multiplicateur de pression permettant d'atteindre 20 kbar. L'enceinte d'essais reçoit d'un côté l'obturateur par lequel arrive le fluide sous pression et de l'autre, les obturateurs et les joints d'essai. Cette enceinte est utilisée pour les essais réalisés à une pression inférieure ou égale à $12 \mathrm{kbar}$. La pression est alors mesurée à l'aide d'une jauge de manganine logée dans la chambre du groupe multiplicateur. Aux pressions supérieures à $12 \mathrm{kbar}$, les joints sont essayés sur un obturateur s'adaptant sur l'enceinte du groupe multiplicateur, à la place de l'obturateur normal. Dans ce cas, la pression est mesurée indirectement à l'aide du manomètre indiquant la pression d'huile du groupe multiplicateur. Ce manomètre a été étalonné préalablement à l'aide de la jauge de manganine. La fiabilité des joints a été testée sous pression d'hélium. Le contrôle de l'étanchéité a été réalisé à l'aide d'un détecteur de fuites $\left({ }^{1}\right)$ et par une mesure de la chute de pression dans l'enceinte entre deux instants déterminés. Un joint est déclaré étanche lorsque ni l'une ni l'autre de ces méthodes ne permettent de détecter une fuite.

3. Partie expérimentale. - L'obturateur conique proposé par les auteurs de la référence [6] permet d'initier l'étanchéité sans qu'il soit nécessaire d'exercer un couple de vissage intense sur la culasse. Cet avantage permet souvent une simplification de la construction. Ces auteurs ont utilisé un empilement de joints de duretés différentes. Nos essais ont montré, cependant

(1) Détecteur Gas Analysis System, Inc, Far Hills New Jersey U.S. A. 


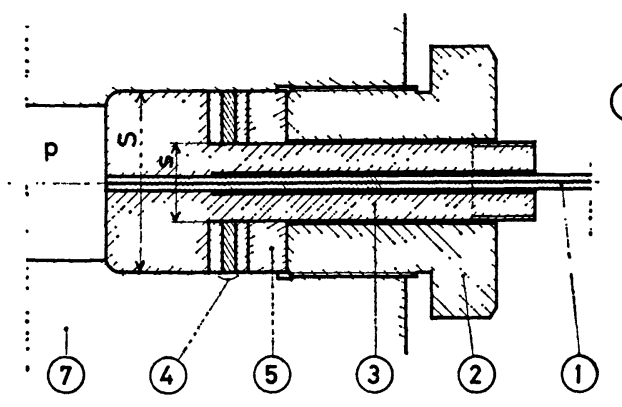

FIG. 1a. - Le fluide sous pression $p$ exerce une force $p S$ sur la tête de l'obturateur. Cette force est transmise aux joints d'aire $(S-s)$. La surpression sur les portées est $p S /(S-s)$.

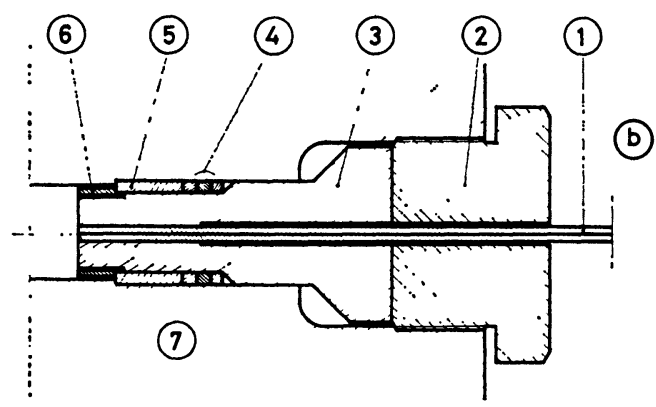

FIG. 1b. - La pression du fluide agit directement sur les joints.

(8)

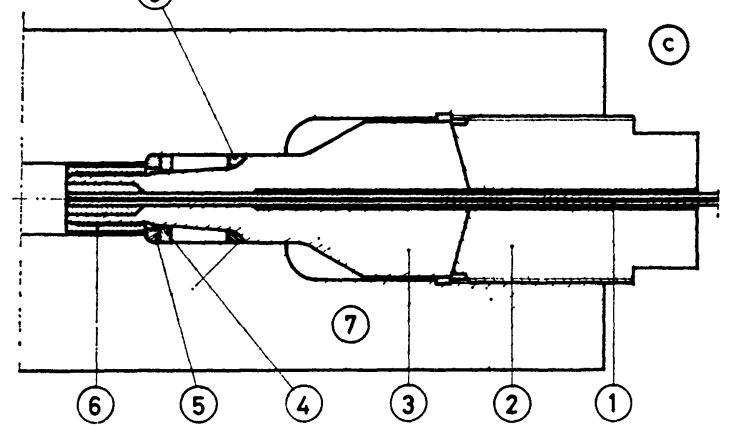

FIG. 1c. - Sous la pression limite, le joint prend la position limite 8.

Fig. 1. - Dispositif d'étanchéité : a) Premier dispositif de Bridgman ; $b$ ) deuxième dispositif de Bridgman ; $c$ ) dispositif de Malfait et Jérôme modifié, à un seul joint. 1 : tube capillaire haute pression, 2 : culasse, 3 : obturateur, $4:$ Joints, 5 : Bague de poussée, 6 : écrou d'extraction, 7 : corps d'enceinte, 8 : position limite $\mathrm{du}$ joint.

qu'un seul suffisait pour obtenir l'étanchéité. Aussi, des joints de différentes matières (acier, cuivre, aluminium, cuivre-béryllium) ont-ils été essayés séparément. Malheureusement, ces joints grippent fortement sur les portées, puisque les couples de frottement sont constitués par des métaux présentant une aptitude particulière aux filiations cristallines. En outre, ils n'assurent plus une étanchéité reproductible dès qu'une certaine valeur, relativement faible de la pression est dépassée. Nous expliquons ceci de la manière suivante : au-delà de cette pression, l'obturateur exerce une réaction axiale importante sur le joint ; celui-ci tend à être repoussé pendant le retour à la pression atmosphérique. Il s'ensuit une diminution de la pression de contact sur les portées, accompagnée du retour élastique des microgéométries des surfaces en contact $[7,8]$; une conductance de fuite s'établit qui rend impossible toute remontée en pression. En revanche, cet inconvénient est supprimé si l'on dépose sur le joint une mince couche d'une substance tendre et plastique. Cette substance doit être choisie pour ses propriétés antigrippantes. Les principaux couples de frottement convenables sont donnés dans la référence [9]. De très bons résultats ont été obtenus avec des joints cadmiés [10]. Les essais montrent cependant qu'au-delà d'une certaine pression que nous appellerons pression limite, l'étanchéité ne peut plus être obtenue. Cette pression dépend de la résistance mécanique du métal de base. Pour des joints de cuivre $\frac{1}{2}$ dur, elle est de $7 \mathrm{kbar}$, tandis que pour des joints de cuivre béryllium $\frac{1}{4}$ écroui non traités, elle est un peu supérieure à $11 \mathrm{kbar}$. Ces pressions limites sont relativement peu élevées ; aussi nous a-t-il paru nécessaire de rechercher le moyen d'accroître leur valeur. L'observation montre que la pression limite correspond à la position limite du joint, c'est-à-dire celle qu'il occupe lorsqu'il s'est écoulé jusqu'à l'extrême limite de la portée (Fig. 1c). Il apparaît que l'étanchéité cesse d'être assurée dès que la vitesse d'écoulement plastique s'annule. Il s'agit donc de trouver un profil retardant le plus possible l'annulation de cette vitesse. Celui qui semble a priori pouvoir apporter la solution est le profil rectiligne. L'utilisation d'obturateurs coniques a confirmé l'accroissement attendu des performances. Toutefois, nous avons observé dans tous les cas, une dissymétrie de l'écoulement plastique des joints, caractérisée par une ondulation de leur ligne moyenne. L'apparition de cette ondulation s'explique ainsi : la symétrie de révolution initiale n'est jamais parfaite; les imperfections d'usinage, les jeux de fonctionnement nécessaires entre les différentes pièces, l'inhomogénéité des matériaux constituant le joint (métal de base, dépôt) font que l'écoulement plastique ne se trouve initié que dans une zone localisée du joint. Aussitôt, le joint perd sa symétrie de révolution. Il en résulte une dissymétrie des forces de pression qui tend à entretenir la dissymétrie de révolution. L'écoulement plastique d'un joint sur une portée conique présente donc un caractère d'instabilité dont les effets ne peuvent aller qu'en s'amplifiant avec l'accroissement de la pression. Ces effets se manifestent essentiellement par l'apparition de contraintes dues à la dissymétrie des forces intérieures (Fig. 2) et par une diminution de la fiabilité du dispositif. Pour remédier à ces inconvénients, l'analyse précédente nous suggère de réduire le plus possible la cause initiale de dissymétrie et d'introduire un effet de contre-réaction capable de stabiliser l'écoulement plastique. Le premier remède est apporté par un centrage aussi précis que possible de l'obturateur dans l'enceinte et le second par l'introduction d'une rupture de pente sur la portée de l'obturateur. Le mécanisme de la régulation de l'écoulement plastique 


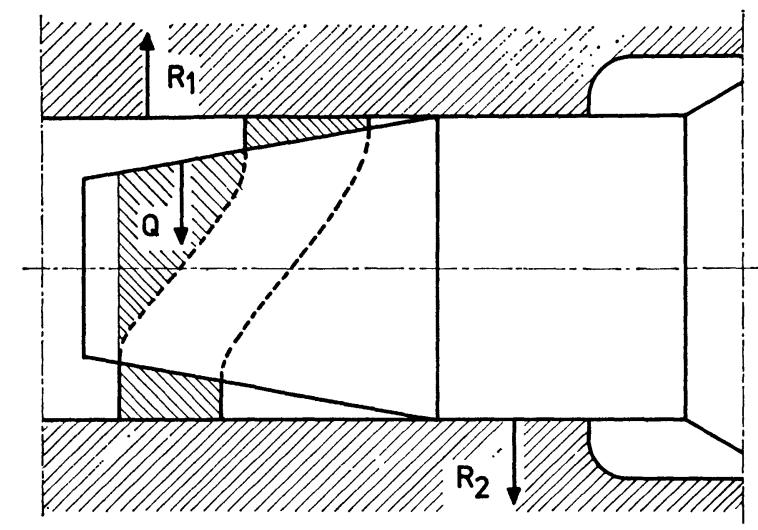

Fig. 2. - Effets de l'écoulement dissymétrique des joints. La dissymétrie de révolution donne naissance aux forces intérieures $Q$ et $R_{1}$ et à la réaction $R_{2}$.

est le suivant : les zones dont les vitesses d'écoulement sont les plus élevées atteignent en premier la rupture de pente. Elles rencontrent une plus grande résistance à l'avancement et dès lors, voient leur vitesse d'écoulement diminuer. Les zones n'ayant pas encore atteint la rupture de pente conservent leur vitesse d'écoulement. Il s'ensuit un rattrapage des premières par les secondes et une restauration rapide de la symétrie de révolution : nous appellerons par la suite partie antérieure et partie postérieure du dispositif, les parties situées de chaque côté de la rupture de pente et rencontrées successivement en parcourant l'axe de l'enceinte de l'intérieur vers l'extérieur. Le dispositif est conçu pour que le joint se répartisse également sur les parties antérieure et postérieure lorsqu'il occupe la position limite. La condition de non-retrait du joint sous l'effet de la réaction de l'obturateur s'écrit

$$
\omega<4 \varphi-\alpha
$$

$\omega$ est le $\frac{1}{2}$ angle au sommet du cône postérieur, $\varphi$ est l'angle de frottement du joint sur les portées, $\alpha$ est le $\frac{1}{2}$ angle au sommet du cône antérieur.

D'après cette relation, la rupture de pente et, par suite, son effet régulateur sont d'autant plus importants que la conicité antérieure est faible. Il est donc recommandé d'opter pour une faible conicité. Toutefois, celle-ci ne doit pas être trop faible car elle rendrait le positionnement initial du joint trop incertain. L'expérience montre que l'adoption d'une conicité de $10 \%$ réalise un bon équilibre entre les deux exigences. L'angle au sommet du cône postérieur ne dépend donc plus que du coefficient de frottement. Celui-ci est fonction de la nature du dépôt réalisé sur les joints. Avec des joints cadmiés, le coefficient de frottement est de 0,07 [11]. Cette valeur fixe l'angle au sommet à $26^{\circ}$. La dernière quantité à préciser pour définir entièrement le dispositif d'étanchéité est l'épaisseur du joint. Celle-ci a été déterminée sur la base des principes suivants :

1) l'épaisseur du joint doit être faible par rapport au diamètre,
2) le joint doit s'écouler sur une distance égale à sa largeur avant d'atteindre la rupture de pente.

Le respect de ces deux principes est une condition nécessaire à l'obtention de bons résultats. Pour les joints expérimentés de diamètre compris entre 13 et $28 \mathrm{~mm}$, nous avons fixé l'épaisseur à $0,9 \mathrm{~mm}$. Cette valeur s'est montrée particulièrement satisfaisante. L'utilisation de joints peu épais présente l'avantage important de permettre $1 \mathrm{a}$ réduction des dimensions extérieures de l'enceinte. En effet comme le diamètre extérieur de l'enceinte et le diamètre intérieur doivent être dans un rapport déterminé $K$, tout gain sur le diamètre du joint se traduit par un gain $K$ fois supérieur sur le diamètre extérieur de l'enceinte. Tous les détails de réalisation relatifs à ce dispositif sont précisés sur la figure 3 .

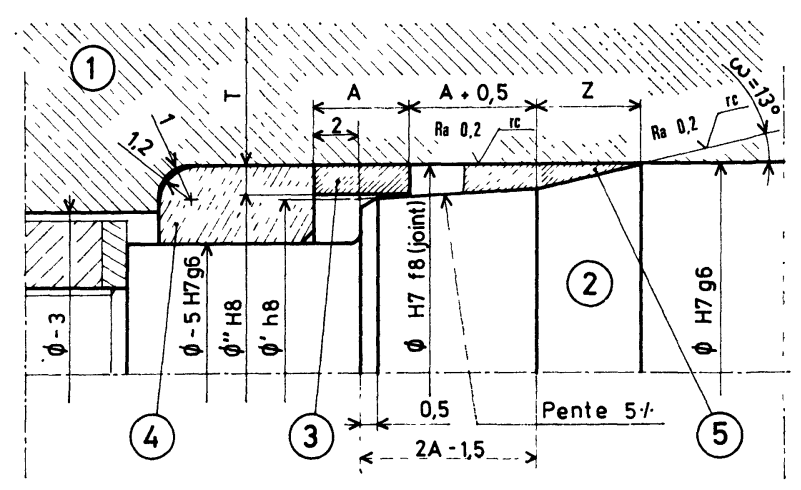

FIG. 3. - Dispositif d'étanchéité à obturateur biconique (détail) : 1 : corps d'enceinte, 2 : obturateur biconique, $3:$ joint d'étanchéité revêtu d'un dépôt électrolytique, $4:$ bague de poussée et de centrage, $5:$ position limite du joint :

$$
\begin{aligned}
T & =0,2309 Z+0,200 \\
A & =\sqrt{(2,3 Z+0,25)^{2}+7 Z^{2}}-(2,3 Z+0,25) \\
\Phi^{\prime} & =\Phi-(0,4618 Z+0,2 A-0,15) \\
\Phi^{\prime \prime} & =\Phi-(0,4618 Z+0,1 A+0,05) .
\end{aligned}
$$

(Les cotes sont exprimées en millimètres)

4. Performances. - Lorsque les déformations de l'enceinte sont faibles, les essais montrent que la pression limite :

1) ne dépend pas du diamètre du joint ;

2) qu'elle décroît avec la dureté du revêtement des joints ;

3) qu'elle est égale à environ trois fois la limite d'élasticité moyenne actuelle du métal de base des joints dans le cas où ceux-ci sont cadmiés.

Les essais montrent également que la fiabilité des joints s'accroît très rapidement lorsque la pression maximale d'utilisation décroît. Les résultats expérimentaux obtenus sont les suivants : la pression limite d'un joint en cuivre $\frac{1}{2}$ dur cadmié est $12 \mathrm{kbar}$, mais à partir de $11 \mathrm{kbar}$, la fiabilité atteint déjà 5 montées. L'étanchéité sous une pression d'hélium de $17 \mathrm{kbar}$ a été obtenue deux fois de suite avec un même joint de 
cuivre béryllium cadmié, le retour à la pression atmosphérique étant réalisé entre les deux montées en pression. Pour ménager l'installation, les essais n'ont pas été poursuivis. Ces deux montées à $17 \mathrm{kbar}$ ne constituent donc pas la performance maximum du dispositif. D'ailleurs, l'examen du joint après essai montre que la pression limite devrait se situer à une valeur notablement supérieure à $17 \mathrm{kbar}$. De plus en choisissant un matériau un peu plus dur que le cuivre béryllium, un acier à $80 \mathrm{kbar}$, de charge à la rupture par exemple, on peut espérer réaliser l'étanchéité à des pressions nettement supérieures à $20 \mathrm{kbar}$. Ce dispositif a été appliqué à la réalisation d'enceintes en alliages légers $[3,12]$, d'une contenance de $3 \mathrm{~cm}^{3}$.

L'une de ces enceintes a été remplie d'hélium sous une pression de 6 kbar puis fermée sur elle-même. Des jauges de contraintes compensées thermiquement, collées sur la paroi externe de l'enceinte permettaient de suivre l'évolution de la pression régnant dans l'enceinte. En 24 heures, on n'a noté aucune modification sensible de l'indication des jauges. En outre, ces enceintes ont été soumises à des essais systématiques de rupture sous pression d'hélium entre $60 \mathrm{~K}$ et $300 \mathrm{~K}$. Ces essais ont démontré la parfaite fiabilité du dispositif d'étanchéité, même à basse température et son bon fonctionnement, malgré les déformations importantes des chambres, peu avant la rupture.

5. Conclusion. - Nous avons mis au point un dispositif d'étanchéité d'une grande fiabilité tant à basse température qu'à température ambiante. Il permet d'atteindre des pressions supérieures à celles qu'autorisent les autres dispositifs connus jusqu'alors. Il s'accommode d'importantes déformations du siège de l'enceinte sous l'effet de la pression. Il ne met en œuvre qu'un seul joint. Ce dispositif ne nécessite qu'un très faible serrage de la culasse pour initier l'étanchéité. Sa compacité permet de réduire à l'extrême les dimensions extérieures de l'enceinte. En outre, il possède tous les avantages des dispositifs dans lesquels la pression agit directement sur les joints ; en particulier, la striction de l'obturateur n'est pas à craindre et le remplacement des joints est aisé. Ce dispositif, ayant déjà fait l'objet de plusieurs applications, a été breveté par l'Anvar [13].

Remerciements. - Je tiens à remercier MM. les Professeurs H. Bartholin et A. Waintal pour les discussions très fructueuses que j'ai eues avec eux ainsi que M. le Professeur D. Bloch pour l'intérêt qu'il a porté à ce travail.

\section{Bibliographie}

[1] Beille, J., Alberts, H. L., Bartholin, H., Bloch, D., VetTiER, C., C. R. Hebd. Séan. Acad. Sci. 275B (1972) 719.

[2] Yelon, W. B., Cox, D. E., Kortman, P. J. and Daniels, W. B., Phys. Rev. B, 9 (1974) 4843.

[3] Vetrier, C., Bloch, D., Paureau, J. and Yelon, D. W., The Fourth International Conference on High Pressure Airapt Kyoto nov. 1974.

[4] Spain, I. L. and Segall, S., Cryogenics (1971).

[5] Bridgman, P. W., Proc. Am. Acad. Arts Sci. 49 (1914) 627.

[6] Malfait, G. et Jerome, D., Revue Phys. Appl. 4 (1969) 467.

[7] Armand, G., Lapujoulade, J. and Paigne, 1961 8th Vacuum
Symposium and 2nd International Congress (Pergamon Press, Oxford) 1962.

[8] Roth, A. and Inbar, A., Vacuum 17 (1967) 5.

[9] CAUBET, J. J., Théorie et pratique industrielle du frottement (Dunod Technip, Paris) 1964.

[10] Vettier, C. and Yelon W. B., J. Phys. Chem. Solids 36 (1975) 401-405.

[11] Paureau, J., à publier.

[12] Paureau, J. et Vettier, C., V International conference on High Pressure and Technology USSR Moscow May 26311975.

[13] Brevet en France no 7433478 du 4.10.1974. 